\title{
Hypodontia - not only an orthodontic problem
}

\section{Hipodoncja - problem nie tylko ortodontyczny}

\author{
Zofia Kielan-Grabowska ${ }^{A-D}$, Beata Kawala ${ }^{\mathrm{E}, \mathrm{F}}$, Joanna Antoszewska-Smith ${ }^{\mathrm{D}-\mathrm{F}}$ \\ Division of Maxillofacial Orthopedics and Orthodontics, Department of Maxillofacial Orthopedics and Orthodontics, Wroclaw Medical University, Poland \\ A - research concept and design; $\mathrm{B}$ - collection and/or assembly of data; $\mathrm{C}$ - data analysis and interpretation; \\ $D$ - writing the article; $\mathrm{E}$ - critical revision of the article; $\mathrm{F}$ - final approval of the article
}

\section{Address for correspondence \\ Zofia Kielan-Grabowska}

E-mail: zosia.kielan@gmail.com

\section{Funding sources}

None declared

Conflict of interest

None declared

Received on January 20, 2019

Reviewed on April 17, 2019

Accepted on June 6, 2019

Published online on December 3, 2019

Cite as

Kielan-Grabowska Z, Kawala B, Antoszewska-Smith J.

Hypodontia - not only an orthodontic problem. Dent Med

Probl. 2019;56(4):373-377. doi:10.17219/dmp/109903

DOI

$10.17219 / \mathrm{dmp} / 109903$

Copyright

○ 2019 by Wroclaw Medical University

This is an article distributed under the terms of the

Creative Commons Attribution 3.0 Unported License (CC BY 3.0)

(https://creativecommons.org/licenses/by/3.0/)

\begin{abstract}
Background. The meta-analysis of the data collected in the years 1939-1993 clearly shows an increasing incidence of hypodontia. The disorder is characterized by a congenital absence of deciduous or permanent teeth. It is a significant esthetical and functional problem, which may negatively affect the patient's psychosocial development.

Objectives. The aim of the study was to determine the scale of hypodontia using a sample of Polish orthodontic patients.

Material and methods. The orthopantomograms and medical records of 674 patients aged $6-15$ years (376 girls and 298 boys) were analyzed in order to identify dental agenesis. Deciduous and wisdom teeth were excluded from the study. Data regarding gender, the location of the disorder as well as the number and type of missing buds was recorded.

Results. Hypodontia was found in $11.6 \%$ of the subjects (7.6\% girls and $4 \%$ boys), more often in the mandible (44.9\%) than in the maxilla (28.2\%) or in both jaws (26.9\%), and these differences were statistically significant $(p=0.096)$. The occurrence rates of uni- and bilateral hypodontia were similar: $51.3 \%$ and $48.7 \%$, respectively. The incidence of left-sided hypodontia (37.2\%) significantly prevailed over rightsided hypodontia (14.1\%) ( $p<0.01)$. Tooth 35 was the most frequently missing one (13.5\%).

Conclusions. The large discrepancy in hypodontia prevalence indicates that geographical differences and varying sample sizes greatly affect the results of studies. The literature lacks comprehensive studies regarding hypodontia in Poland. More studies with similar sample sizes and selection criteria need to be carried out. Through an early detection of hypodontia, it is possible to plan comprehensive, interdisciplinary treatment. Regardless of the discrepancies in the literature as to the exact location of the problem, hypodontia affects over $10 \%$ of the population of patients treated orthodontically, which justifies the need to study this issue.
\end{abstract}

Key words: hypodontia, dental agenesis, oligodontia

Słowa kluczowe: hipodoncja, agenezja zęó́w, oligodoncja 


\section{Introduction}

The meta-analysis of the data collected in the years 1939-1993 clearly points to an increase in the prevalence of missing teeth, ${ }^{1}$ including hypodontia. This disorder, also referred to as dental agenesis, is the most common developmental irregularity affecting human teeth ${ }^{2}$ and is characterized by a congenital absence of deciduous or permanent teeth. The term 'hypodontia' is usually used to describe the absence of 1-6 tooth buds, which distinguishes it from oligodontia - a systemic absence of tooth buds. In permanent dentition, the most commonly missing teeth (excluding third molars) are either lower second premolars or upper lateral incisors. ${ }^{2} \mathrm{Hy}$ podontia is often accompanied by microdontia, delays in tooth development, ectopic tooth positioning, taurodontism, shortened roots, or enamel hypoplasia. Having fewer teeth is a significant esthetic and functional problem, and it can severely impact one's mental and social development.

The etiology of hypodontia involves multiple factors and is therefore complex. The patient's genetic background is considered to be the dominant factor ${ }^{2-7}$; this is proven by the fact that hypodontia has frequently been reported in twins (more frequently in monozygotic than in dizygotic cases) and in successive generations, in the form of genetically conditioned defects (ectodermal dysplasia) or congenital defects (Down's syndrome).

Environmental factors, such as diseases the mother suffered from during pregnancy (including viral and contagious diseases), poor nutrition, alcohol consumption, certain drugs (e.g., anticonvulsants), and past injuries, can also contribute to dental agenesis. In the postnatal period, tooth buds can be negatively affected by chemoand radiotherapy being part of cancer treatment. ${ }^{2-4,6,7} \mathrm{Re}-$ garding the absence of specific tooth buds, reports most commonly indicate the absence of second molars with simultaneous sella turcica calcification, and the absence of the second premolar and lateral incisor buds as a result of an innervation defect which occurred at the last stage of tooth bud development (neurogenic theory). ${ }^{2}$

Such a complex etiology of hypodontia requires any treatment applied to be highly specialized. Depending on the patient's age, the location of missing teeth and any coexistent malocclusion, the patient must obtain an individualized treatment plan aimed at recreating the correct function and tooth esthetics, and at preventing complications of hypodontia, such as periodontal damage, the lack of alveolar growth or the development of malocclusion. The 2 main therapeutic options are the orthodontic closure of the space, and maintaining or recreating the space for future insertion.

In the frontal segment, the most proficient method - in terms of esthetics - is to mesialize a canine into the place of a missing lateral incisor. This method uses the patient's own teeth to close the gap. It is also minimally invasive and produces a relatively fast result, although it entails the need to change the shape and color of canines to make them resemble incisors. Furthermore, this method can distort canine guidance and the smile line.

The other treatment option - frontal dental implants comes with even more issues. One must remember that the implantation procedure can be performed only after the jaw growth period is complete; until then, removable dentures or bridgeworks can be used. Furthermore, the bone surface under such dentures can disappear over time, which additionally limits the implantation possibilities. $^{2,8}$ The use of implants can be beneficial, but in the lateral section. Restrictions such as insufficient space and the proximity of important anatomical structures in this area should be taken into account, though. ${ }^{2,8}$

Due to its multi-factor etiology and treatment difficulties, hypodontia should not be ignored. Investigating the prevalence of hypodontia is essential in terms of early diagnosis and treatment planning; thus, it should be closely monitored, starting with the necessary basics, i.e., epidemiology.

The aim of the study was to determine the current prevalence and distribution of hypodontia in permanent dentition (excluding third molars) in Polish orthodontic patients, and to compare the results with other international studies.

\section{Material and methods}

Initially, one clinician (Z.K.-G.) examined 2,000 patients and their orthopantomograms as they arrived at the Department of Maxillofacial Orthopedics and Orthodontics of Wroclaw Medical University, Poland, between 2014 and 2018, to identify congenital missing teeth. All permanent teeth were investigated apart from third molars. A tooth was diagnosed as congenitally missing if the mineralization of its crown could not be recognized. If a clear diagnosis of hypodontia could not be made, the images were excluded from the study. Other exclusion criteria were developmental defects (craniofacial syndromes, or cleft lips and palates), a loss of permanent teeth caused by injury or decay, and extractions due to orthodontic indications.

The study group consisted of 674 panoramic radiographs of children and adolescents aged 6-15 years (376 girls and 298 boys). Data such as gender, the location of the disorder, and the number and type of missing teeth buds were also recorded.

The statistical analysis was carried out with the IBM SPSS Statistics for Windows software, v. 25 (IBM Corp., Armonk, USA). The package was also used to carry out the Shapiro-Wilk tests, $\chi^{2}$ independence tests and Student's $t$-tests for independent samples. The significance level was assumed to be $p=0.05$, and $0.05<p<0.1$ was considered statistically significant. 


\section{Results}

A total of 674 records were analyzed, including 376 girls and 298 boys. The results of the analysis of hypodontia occurrence, broken down by gender, are presented in Table 1 . Hypodontia was found in $11.6 \%$ of the subjects $(\mathrm{n}=78 / 674) ; 7.6 \%$ were girls $(\mathrm{n}=51 / 674)$ and $4 \%$ were boys $(\mathrm{n}=27 / 674)$. The $X^{2}$ independence analysis indicated that both variables were interrelated at the level of statistical tendency only $(p=0.07)$.

At the next stage of the study, the 3 nominal variables were summarized using the frequency analysis. The absence of teeth was found more often in the lower jaw $(\mathrm{n}=35 / 78)$ than in the upper jaw $(\mathrm{n}=22 / 78)$ or simultaneously in both jaws $(n=21 / 78)$ (Table 2$)$. In terms of percentages compared, the difference was significant only at the level of statistical tendency $(p=0.096)$.

The occurrence rates of unilateral hypodontia $(\mathrm{n}=40 / 78)$ and bilateral hypodontia $(\mathrm{n}=38 / 78)$ were similar (Table 3). The difference in the compared percentages was not statistically significant $(p=0.736)$.

In the examined material, a greater number of patients were diagnosed with left-sided hypodontia $(n=29 / 78)$ as compared to the right-sided hypodontia cases $(n=11 / 78)$ (Table 4). The difference in the compared percentages was statistically significant $(p=0.010)$.

The percentage share of particular tooth types in hypodontia is presented in Table 5 . The most frequently missing tooth was 35 (13.5\%). The second most frequently missing tooth was $12(12.4 \%)$, followed by tooth 22 (11.2\%), 45 (10.6\%), 15 (10\%), and $25(9.4 \%)$.

Table 1. Statistical analysis of the prevalence of hypodontia in boys and girls

\begin{tabular}{|l|c|c|c|c|}
\hline \multicolumn{1}{|c|}{ Gender } & $n(\%)$ & $x^{2}$ & $p$-value & Yule's $\varphi$ \\
\hline Boys & $27(9.1)$ & & & \\
Girls & $51(13.6)$ & 3.3 & 0.070 & 0.070 \\
\hline
\end{tabular}

Table 2. Statistical analysis of the prevalence of hypodontia in the maxilla and/or the mandible

\begin{tabular}{|l|c|c|c|c|}
\hline \multicolumn{1}{|c|}{ Jaw } & $\mathrm{n}(\%)$ & $\begin{array}{c}\text { Percentage of valid } \\
\text { observations [\%] }\end{array}$ & $x^{2}$ & $p$-value \\
\hline $\begin{array}{l}\text { Mandible } \\
\text { Maxilla }\end{array}$ & $22(5.2)$ & 44.9 & & \\
$\begin{array}{l}\text { Both } \\
\text { (simultaneous occurrence) }\end{array}$ & $21(3.1)$ & 28.2 & 4.69 & 0.096 \\
\hline
\end{tabular}

Table 3. Statistical analysis of the prevalence of hypodontia in relation to the number of sides affected

\begin{tabular}{|l|c|c|c|c|}
\hline $\begin{array}{c}\text { Number of sides } \\
\text { affected }\end{array}$ & $n(\%)$ & $\begin{array}{c}\text { Percentage of valid } \\
\text { observations [\%] }\end{array}$ & $x^{2}$ & $p$-value \\
\hline 1 (unilateral hypodontia) & $40(5.9)$ & 51.3 & 0.11 & 0.738 \\
\hline 2 (bilateral hypodontia) & $38(5.6)$ & 48.7 & & \\
\hline
\end{tabular}

Table 4. Statistical analysis of the prevalence of hypodontia in relation to the side affected

\begin{tabular}{|l|c|c|c|c|}
\multicolumn{1}{|c|}{ Side affected } & $n(\%)$ & $\begin{array}{c}\text { Percentage of valid } \\
\text { observations [\%] }\end{array}$ & $x^{2}$ & $p$-value \\
\hline $\begin{array}{l}\text { Right } \\
\text { Left }\end{array}$ & $29(11.6)$ & 14.1 & & \\
$\begin{array}{l}\text { Both } \\
\text { (simultaneous occurrence) }\end{array}$ & $38(5.6)$ & 47.2 & 14.54 & 0.010 \\
\hline
\end{tabular}

Table 5. Percentage share of missing teeth in hypodontia

\begin{tabular}{|c|c|c|c|}
\hline $\begin{array}{c}\text { Tooth } \\
\text { number }\end{array}$ & $\begin{array}{l}\text { Number } \\
\text { of missing } \\
\text { tooth buds }\end{array}$ & $\begin{array}{c}\text { Percentage of all } \\
\text { missing tooth buds } \\
{[\%]}\end{array}$ & $\begin{array}{c}\text { Percentage of individuals } \\
\text { with hypodontia } \\
{[\%]}\end{array}$ \\
\hline 12 & 21 & 12.4 & 26.9 \\
\hline 13 & 1 & 0.6 & 1.3 \\
\hline 14 & 3 & 1.8 & 3.8 \\
\hline 15 & 17 & 10.0 & 21.8 \\
\hline 17 & 2 & 1.2 & 2.6 \\
\hline 22 & 19 & 11.2 & 24.4 \\
\hline 23 & 1 & 0.6 & 1.3 \\
\hline 24 & 2 & 1.2 & 2.6 \\
\hline 25 & 16 & 9.4 & 20.5 \\
\hline 27 & 3 & 1.8 & 3.8 \\
\hline 31 & 11 & 6.5 & 14.1 \\
\hline 32 & 3 & 1.8 & 3.8 \\
\hline 33 & 4 & 2.4 & 5.1 \\
\hline 34 & 1 & 0.6 & 1.3 \\
\hline 35 & 23 & 13.5 & 29.5 \\
\hline 37 & 7 & 4.1 & 9.0 \\
\hline 41 & 4 & 2.4 & 5.1 \\
\hline 42 & 4 & 2.4 & 5.1 \\
\hline 43 & 3 & 1.8 & 3.8 \\
\hline 44 & 1 & 0.6 & 1.3 \\
\hline 45 & 18 & 10.6 & 23.1 \\
\hline 47 & 6 & 3.5 & 7.7 \\
\hline Total & 170 & 100.0 & 217.9 \\
\hline
\end{tabular}

\section{Discussion}

The analysis of the prevalence of dental agenesis among the Hutterite population of western Canada, isolated in religious and genetic terms, brought the result of approx. $47 \% .^{9}$ However, the findings of the contemporary literature prove that the occurrence of hypodontia varies in different populations. According to the meta-analysis carried out by Khalaf et al., the prevalence of hypodontia is $13.4 \%$ for Africa, $7 \%$ for Europe, 6.3\% for Asia, 6.3\% for Australia, 5\% for North America, and $4.4 \%$ for Latin America and the Caribbean. ${ }^{10}$ This study confirms the results of an earlier systematic review, according to which dental agenesis is less frequent in North America than in Europe or Australia. ${ }^{11}$ 
Our finding (11.6\%) is almost twice as high as that provided by Khalaf et al., and is also higher than that reported in Italian studies (Lo Muzio et al. - 5.17\%, Polastri et al. - $5.14 \%$ and Gracco et al. - 9\%), ${ }^{12-14}$ Turkish studies (Celikoglu et al. $-4.6 \%$ and Sisman et al. $-7.54 \%)^{15,16}$ or in an Iranian study (Amini et al. - 5.21\%). ${ }^{17}$ However, it is similar to the results of an analysis of hypodontia occurrence among the Korean population (Chung et al. $-11.2 \%)^{18}$ and is even lower than the results obtained in a study carried out on German children (Behr et al. - 12.6\%), ${ }^{19}$ not to mention other results found in the Polish literature, where - depending on the group analyzed - the rate of hypodontia occurrence ranged from $9.5 \%^{6}$ to as much as $14.8 \% .{ }^{20}$ Such data only stresses the extent of the problem of hypodontia in Asia and Europe.

Regarding the relationship between hypodontia and gender, the majority of studies indicate a higher occurrence rate among women, as in our study. ${ }^{5,14}$ In women, the phenomenon was observed 1.22 times $^{10}$ to 1.37 times $^{11}$ more often than among the male population of the same race. The more frequent occurrence of missing teeth among girls is also confirmed by a Japanese study $(10.8 \%$ in girls and $8.7 \%$ in boys ${ }^{21}$ and by a Polish study held in Cracow (10.8\% in girls and $7.3 \%$ in boys). ${ }^{6}$ However, this may be due to the fact that women seek dental care more often than men.

Our finding that dental agenesis is statistically more frequent in the lower jaw than in the upper jaw contradicts the results of other studies, according to which missing teeth is more often diagnosed in the upper arch., $514,22,23$

When it comes to the relationship between hypodontia and the quadrant of dentition, one Italian study confirms the results of our study, indicating similar occurrence rates of unilateral and bilateral hypodontia, ${ }^{14}$ whereas a Turkish study contradicts these findings, instead indicating that unilateral hypodontia is more frequent $(68.7 \%){ }^{16}$

As for the occurrence of hypodontia in the left or right side, no significant differences were indicated among the Iranian population ${ }^{17}$ or among the Turkish population, ${ }^{16}$ which differs from our results, where left-sided hypodontia was more frequently found than right-sided hypodontia.

The literature displays a certain inconsistency concerning the most frequently missing teeth. In the European population, agenesis more often affects teeth 35 and 45, 12 and 22 , and 15 and $25,{ }^{10}$ as confirmed by the results of our study. According to Mattheeuws et al., Polder et al., Lo Muzio et al., and Gracco et al., the most frequently missing tooth is the second premolar of the lower jaw. ${ }^{1,11,12,14}$ In turn, Iranian, Brazilian and American studies found that the lateral incisor of the upper jaw was the most frequently missing tooth. ${ }^{17,22,24}$ All of these studies point to a certain interdependence - agenesis most often affects the distal tooth from a given group.

We are aware that the present results were obtained by examining only individuals visiting the orthodontist. On the other hand, orthopantomograms are a routine diagnostic tool for the orthodontist, because who else can make diagnosis, for example, before cross bite treatment at an early age (absolute indication), and at the same time detect tooth agenesis in the maxilla, which may accompany class III malocclusion? Therefore, as orthodontists, we feel entitled and obliged to detect hypodontia, and thus to provide objective, evidence-based results of epidemiological research on this issue.

The unification of study groups is another limitation, but it does not apply only to our research, but to all of the abovementioned. It must be remembered that the more research is conducted, the easier it is to employ retrospective criticism, and to develop the criteria for optimal material and methods for further studies.

The age of patients in the study is the last limitation. The panoramic radiographs of patients younger than 9 years old should be retaken over time to prevent the classification of late mineralized teeth as congenitally missing and to confirm a diagnosis of hypodontia.

\section{Conclusions}

The large discrepancy in hypodontia prevalence observed in all of the above studies indicates that geographical differences as well as different sample sizes greatly affect the research results. The literature lacks comprehensive studies regarding dental agenesis among the Polish population. More studies with the same sample size and selection criteria need to be carried out in order to gain an actual view on hypodontia.

Thanks to an early detection of hypodontia, it is now possible to plan comprehensive, interdisciplinary treatment to ensure correct functioning and esthetics. Regardless of the discrepancies in the literature as to the exact location of the problem, hypodontia affects over $10 \%$ of the population of patients receiving orthodontic treatment, which justifies the need to study the issue of congenital tooth absence.

\section{ORCID iDs}

Zofia Kielan-Grabowska (D) https://orcid.org/0000-0003-3199-3335 Beata Kawala (D) https://orcid.org/0000-0001-8453-1076

Joanna Antoszewska-Smith (i) https://orcid.org/0000-0001-9192-1064

\section{References}

1. Mattheeuws N, Dermaut L, Martens G. Has hypodontia increased in Caucasians during the 20th century? A meta-analysis. Eur J Orthod. 2004;26(1):99-103.

2. Jędryszek A, Kmiecik M, Paszkiewicz A. Review of modern knowledge on hypodontia [in Polish]. Dent Med Probl. 2009;46(1):118-125.

3. Biedziak B. Aetiology and occurence of tooth agenesis - review of the literature [in Polish]. Dent Med Probl. 2004;41(3):531-535.

4. Rakhshan V. Congenitally missing teeth (hypodontia): A review of the literature concerning the etiology, prevalence, risk factors, patterns and treatment. Dent Res J. 2015;12(1):1-13.

5. Fekonja A. Hypodontia in orthodontically treated children. Eur J Orthod. 2005;27(5):457-460. 
6. Dyras M, Jankowska K, Czupryna S. The estimation of the development disorders prevalence in patients treated in the Orthodontic Department of the Stomatological Institute in Collegium Medicum Jagiellonian University of Cracow [in Polish]. Dent Med Probl. 2003;40(2):349-354.

7. Gregorczyk-Maga I, Merta U, Kołodziej I, Jurczak A, Wiśniewska G. Prosthetic methods of hypodontia treatment on the basis of the literature [in Polish]. Nowa Stomatol. 2013;18(4):171-174.

8. Grotowski TA. Implant-based prosthesis in cases of hypodontia of upper lateral incisors. Long term observations [in Polish]. Magazyn Stomatol. 2006;16(4):50-57.

9. Mahaney MC, Fujiwara TM, Morgan K. Dental agenesis in the Dariusleut Hutterite Brethren: Comparisons to selected Caucasoid population surveys. Am J Phys Anthropol. 1990;82(2):165-177.

10. Khalaf K, Miskelly J, Voge E, Macfarlane TV. Prevalence of hypodontia and associated factors: A systematic review and meta-analysis. J Orthod. 2014;41(4):299-316.

11. Polder BJ, Van't Hof MA, Van der Linden FP, Kuijpers-Jagtman AM. A meta-analysis of the prevalence of dental agenesis of permanent teeth. Community Dent Oral Epidemiol. 2004;32(3):217-226.

12. Lo Muzio L, Mignogna MD, Bucci P, Sorrentino F. Statistical study of the incidence of agenesis in a sample of 1,529 subjects [in Italian]. Minerva Stomatol. 1989;38(9):1045-1051.

13. Polastri F, Cerato E, Gallesio C. The clinico-radiological assessment of dental anomalies with real and apparent numerical defects [in Italian]. Minerva Stomatol. 1991;40(6):415-423.

14. Gracco ALT, Zanatta S, Forin Valvecchi F, Bignotti D, Perri A, Baciliero F. Prevalence of dental agenesis in a sample of Italian orthodontic patients: An epidemiological study. Prog Orthod. 2017;18:33.

15. Celikoglu M, Kazanci F, Miloglu O, Oztek O, Kamak H, Ceylan I. Frequency and characteristics of tooth agenesis among an orthodontic patient population. Med Oral Patol Oral Cir Bucal. 2010;15(5):e797-e801.

16. Sisman Y, Uysal T, Gelgor IE. Hypodontia. Does the prevalence and distribution pattern differ in orthodontic patients? Eur J Dent. 2007;1(3):167-173.

17. Amini F, Rakhshan V, Babaei P. Prevalence and pattern of hypodontia in the permanent dentition of 3,374 Iranian orthodontic patients. Dent Res J (Isfahan). 2012;9(3):245-250.

18. Chung $\mathrm{CJ}, \mathrm{Han} \mathrm{JH}, \mathrm{Kim} \mathrm{KH}$. The pattern and prevalence of hypodontia in Koreans. Oral Dis. 2008;14(7):620-625.

19. Behr $M$, Proff $P$, Leitzmann $M$, et al. Survey of congenitally missing teeth in orthodontic patients in Eastern Bavaria. Eur J Orthod. 2011;33(1):32-36.

20. Grzesiewska K, Hornik E, Pisarska H. Lack of permanent tooth buds in connection with malocclusion of patients treated in the Orthodontic Department of Silesian Medical Academy [in Polish]. Czas Stomat. 1978;21(5):307-309.

21. Goya HA, Tanaka S, Maeda T, Akimoto Y. An orthopantomographic study of hypodontia in permanent teeth of Japanese pediatric patients. J Oral Sci. 2008;50(2):143-150.

22. Gomes RR, da Fonseca JA, Paula LM, Faber J, Acevedo AC. Prevalence of hypodontia in orthodontic patients in Brasilia, Brazil. Eur J Orthod. 2010;32(3):302-306.

23. Wong AT, McMillan AS, McGrath C. Oral health-related quality of life and severe hypodontia. J Oral Rehabil. 2006;33(12):869-873.

24. Muller TP, Hill IN, Peterson AC, Blayney JR. A survey of congenitally missing permanent teeth. J Am Dent Assoc. 1970;81(1):101-107. 